\title{
Using (3+1)D Space to Investigate a Modulated Superstructure Mystery Jeffrey Lovelace ${ }^{1}$, Gloria Borgstahl ${ }^{2}$ \\ ${ }^{1}$ Univ. of Nebraska Medical Center ${ }^{2}$ The Eppley Inst For Cancer Res \\ jlovelace@unmc.edu
}

Two commensurately modulated structures (PDB entries 4n3e (Sliwiak et al. 2015) and 6sjj (Smietanska et al. 2020)) were solved using translational noncrystallographic symmetry (tNCS). The data required the use of large supercells, sevenfold and ninefold, respectively, to properly index the reflections. Commensurately modulated structures can be challenging to solve. Molecular-replacement software such as Phaser can detect tNCS and either handle it automatically or, for more challenging situations, allow the user to enter a tNCS vector, which the software then uses to place the components. Although this approach has been successful in solving these types of challenging structures, it does not make it easy to understand the underlying modulation in the structure or how these two superstructures are related. The mystery was how could a slight change in crystallization condition have resulted in the observed large change in the size of the superstructure (from $7 \mathrm{x}$ to $9 \mathrm{x}$ ) with no significantly observed large changes in the chains composing the superstructure. A way to investigate this problem is to view the atoms and associated parameters as following periodic atomic modulation functions (AMFs) in higher dimensional space, and what is being observed in these superstructures are the points where these higher dimensional AMFs intersect physical 3D space (Lovelace \& Borgstahl, 2021). In this case, although the two 3D structures, with a sevenfold and a ninefold superstructure, seem to be quite different, describing those structures within the higher dimensional superspace approach makes a strong case that they are closely related, as they show very similar AMFs and can be described with one unique $(3+1) \mathrm{D}$ structure. With this approach, the addition of the small molecule caused only a small change (q Vector) to the intersection of the (3+1)D structure with 3D space. We find the modulation is easier to understand, especially when represented as an animated gif (Fig. 1).

References

Lovelace, J. J. \& Borgstahl, G. E. O. (2021). Acta Crystallographica Section D 77.

Sliwiak, J. Dauter, Z. Kowiel, M. McCoy, A. J. Read, R. J. \& Jaskolski, M. (2015). Acta Crystallogr D Biol Crystallogr 71, 829843.

Smietanska, J. Sliwiak, J. Gilski, M. Dauter, Z. Strzalka, R. Wolny, J. \& Jaskolski, M. (2020). Acta Crystallographica Section D 76, 653-667.

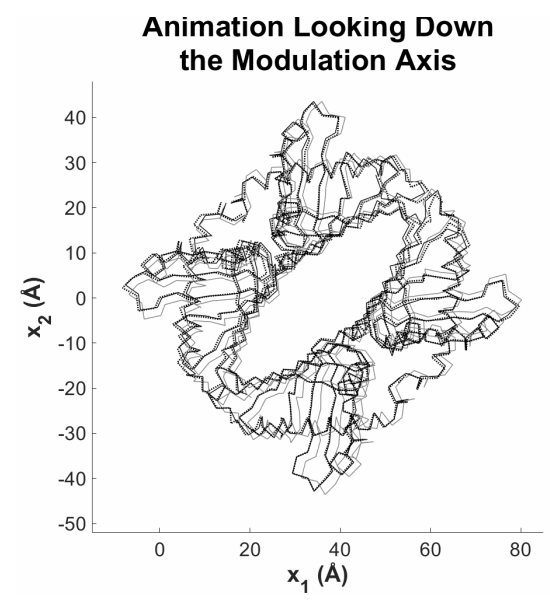

Figure 1 